\title{
Perioperative Landiolol Infusion Reduces the Incidence of Atrial Fibrillation after Pulmonary Lobectomy: Postoperative Randomized Controlled Study
}

\author{
Kaori Yagi' ${ }^{1}$, Jitsuo Usuda², Atsuhiro Sakamoto ${ }^{1}$ \\ ${ }^{1}$ Department of Anesthesiology, Nippon Medical School Hospital, Tokyo, Japan \\ ${ }^{2}$ Department of Thoracic Surgery, Nippon Medical School Hospital, Tokyo, Japan \\ Email: fuku-kaori@nms.ac.jp
}

Received 21 July 2016; accepted 23 August 2016; published 26 August 2016

Copyright (C) 2016 by authors and Scientific Research Publishing Inc.

This work is licensed under the Creative Commons Attribution International License (CC BY).

http://creativecommons.org/licenses/by/4.0/

(c) (i) Open Access

\section{Abstract}

Background and Objective: Atrial fibrillation is a common complication after lung resection. We sought to determine the relationship between low-dose landiolol only intraoperatively administration and the incidence of atrial fibrillation development in patients who did not have atrial fibrillation before undergoing lung resection. Methods: Forty-five patients undergoing lung resection (lobectomy or bilobectomy), as indicated for lung cancer at Nippon Medical Hospital, between August 2012 and September 2013. Two patients were excluded from the final analysis. Patients were given either intravenous landiolol $(n=22)$ or placebo $(n=21)$ during lobectomy or bilobectomy only intraoperatively. This is prospective, randomized, placebo-controlled study. Main Outcome Measures: The primary end point was the incidence of sustained atrial fibrillation ( $\geq 30$ min). Results: Postoperative atrial fibrillation occurred in $1(4.5 \%)$ of the 22 patients in the landiolol group and $6(28.6 \%)$ of the 21 patients in the placebo group. No serious adverse effects such as bradycardia and hypotention secondary to landiolol were observed. Conclusion: Low-dose landiolol infusion intraoperatively reduced the incidence of clinically significant atrial fibrillation in patients undergoing pulmonary lobectomy.

\section{Keywords}

Atrial Fibrillation, Lung Resection, Lobectomy, Bilobectomy, Landiolol

\section{Introduction}

Atrial Fibrillation (AF) is one of the most common complications after thoracic surgery. In addition to reducing

How to cite this paper: Yagi, K., Usuda, J. and Sakamoto, A. (2016) Perioperative Landiolol Infusion Reduces the Incidence of Atrial Fibrillation after Pulmonary Lobectomy: Postoperative Randomized Controlled Study. Open Journal of Anesthesiology, 6, 119-123. http://dx.doi.org/10.4236/ojanes.2016.68020 
quality of life and causing palpitations and cardiac hypofunction, AF also increases the risk of cardiogenic embolus. Therefore, it is necessary to prevent the development of AF in patients undergoing thoracic surgery.

AF often occurs after cardiac surgery. For this reason, various studies have investigated means of inhibiting AF after cardiac surgery. One such study used landiolol. This study, the JL-NIGHT study, demonstrated that intraoperative administration of landiolol hydrochloride reduces the incidence of AF after open-heart surgery [1]. On the other hand, it has also been noted that there is an elevated risk of AF after lung resection, with incidences of approximately $4 \%-30 \%$ in patients undergoing lobectomy and $10 \%-40 \%$ in patients undergoing pneumonectomy [2]-[4]. Although the efficacy of landiolol administration has been investigated in for lung surgeries, more studies have examined its use for heart surgeries. Further, while several studies have reported the efficacy of landiolol [5], no prospective study has investigated the effects of only intraoperative low-dose landiolol infusion in patients undergoing lung resection. Therefore, we undertook a prospective study to determine whether administration of low-dose landiolol could reduce the incidence of AF after lobectomy.

\section{Methods}

\subsection{Patients}

The study protocol was registered with the University Hospital Medical Information Network (UMIN) Clinical Trials Registry (UMIN000007561). Ethical approval for this study (Protocol number 223009) was provided by the Institutional Review Board of Nippon Medical School Hospital, Tokyo, Japan, (Chairperson: Hospital director Sakamoto) on August 8, 2011. We obtained written informed consent from all patients on the day before surgery. This study included 45 patients undergoing lung resection at Nippon Medical School Hospital between August 2012 and September 2013. The indication for lung resection in all subjects was lung cancer, which required treatment with lobectomy or bilobectomy. The patients were randomized to receive landiolol (2.5 $\mu \mathrm{g} \cdot \mathrm{kg}^{-1} \cdot \mathrm{min}^{-1}$ ) (Ono Pharmaceutical Co., Osaka, Japan), or an identical amount of saline as a placebo. Both landiolol and the placebo were administered, intravenously during anesthesia. All patients had an American Society of Anesthesiologists (ASA) physical status of I/II. The exclusion criteria were acute myocardial infarction within 3 days prior to surgery, a history of supraventricular arrhythmia requiring treatment, sinus node disease, presence of a permanent pacemaker, severe heart failure (New York Heart Association III/IV or ejection fraction $<35 \%$ ), atrioventricular block (AV block $\geq$ second degree), contraindications to $\beta$-blocker therapy, secondary AF with a known primary cause (e.g., electrolyte imbalance, Wolff-Parkinson-White syndrome or hyperthyroidism), hypotension ( $<90 / 60 \mathrm{mmHg}$ ), and the perioperative use of an anti-arrhythmic agent other than digitalis.

\subsection{Study Protocol}

At enrolment in the study, the patients were randomized using the sealed envelope method to receive either the placebo or the study drug. Patients were continuously monitored from immediately after surgery for up to 72 hours thereafter. Landiolol or saline was administered from the start of anesthesia as a continuous infusion at an initial rate of $2.5 \mu \mathrm{g} \cdot \mathrm{kg}^{-1} \cdot \mathrm{min}^{-1}$, which was titrated to a maximum rate of $20 \mu \mathrm{g} \cdot \mathrm{kg}^{-1} \cdot \mathrm{min}^{-1}$ (reassessed every 10 $\mathrm{min}$ ) based on the hemodynamic or electrocardiographic response. The maintenance infusion of landiolol was titrated upward to maintain the ventricular rate at $<90$ beats/min. If hypotension (systolic blood pressure $<90$ beats/min) or bradycardia (heart rate $<50$ beats/min) occurred, the dose was reduced or infusion of the drug was discontinued until symptoms resolved.

The Holter electrocardiogram (ECG) was recorded on a secure digital (SD) card-type recorder (Fukuda Denshi 600; Fukuda Denshi Co., Tokyo, Japan). Postoperative recording was initiated after the surgery and continued for 72 hours.

The occurrence of episodes of AF lasting more than 30 minutes was considered as the study endpoint. More specifically, the primary endpoint was the frequency of AF occurrence from the end of the surgery to 72 hours thereafter.

Age, height, and weight were recorded for each patient. Information was also collected on preoperative pulmonary status, which was expressed as the percentage of the predicted value of forced vital capacity.

\subsection{Statistical Analysis}

Continuous variables are expressed as mean \pm standard deviation (SD). Clinical characteristics of the two groups 
were compared using the independent t-test for continuous variables and the chi-square test for categorical variables. Adverse events were analyzed by the chi-square test, as appropriate. In all analyses, $\mathrm{P}<0.05$ was considered statistically significant.

\section{Results}

One patient was excluded because the surgery type was changed intraoperatively, and another was excluded because of undergoing reoperation; hence, 43 patients completed the study. There were no statistically significant differences between the two groups in terms of sex, age, height, weight, and other preoperative data or the measured lung function parameters (Table 1). Further, no statistically significant differences were found in terms of operation time, anesthesia time, or the type of operation. However, no bilobectomy was performed in the landiolol group.

The numbers of patients with arrhythmias are provided (Table 2). AF developed in $17 \%$ of the patients overall, including $5 \%$ of patients in the landiolol group and $28 \%$ of patients in the control group $(\mathrm{P}=0.03$, chi-square test). No differences were found between the two groups in terms of the incidences of other types of arrhythmias. AF developed postoperatively on day 2 in 2 patients and on day 3 in 4 patients. Episodes of AF occurred only once in the patients in whom it was observed. Patients in whom AF occurred were not treated for it immediately because the Holter ECG was not analyzed instantly and they had no subjective symptoms. There was no difference between the landiolol and control groups with regard to heart rate. Indeed, there were no significant differences in heart rate between the two groups throughout the Holter monitoring period (Table 3). AF was not observed intraoperatively in any patient.

With respect to side effects, hypotension, bradycardiaand other serious eventswere not observed in either group.

\section{Table 1. Epidemiological data on the study cohort.}

\begin{tabular}{cccc}
\hline & Landiolol & Placebo & P \\
\hline No. of patients & 22 & 21 & 0.12 \\
Perioperative data & & & 0.34 \\
Gender (male) & 9 & $69.1 \pm 7.10$ & 0.29 \\
Age (years) & $70.9 \pm 7.54$ & $158 \pm 9.8$ & 0.21 \\
Height (cm) & $156 \pm 8.3$ & $55 \pm 10.4$ & 0.30 \\
Weight (kg) & $51.9 \pm 7.3$ & $73.3 \pm 9.30$ & 0.24 \\
FEV1\% & $70.6 \pm 4.96$ & $110 \pm 18.9$ & 0.16 \\
VC & $119 \pm 25.5$ & & 0.80 \\
Perioperative data & & $228 \pm 78.5$ & $331 \pm 101$ \\
Bilobectomy & $212 \pm 86.8$ & & 0.52 \\
Anesthesia time (min) & $323 \pm 110$ & & 0.30 \\
Operation time (min) & & & \\
\hline
\end{tabular}

FEV1\%: forced expiratory volume in $1 \mathrm{~s}$; VC: vital capacity; Values are mean \pm SD numbers.

Table 2. Results of heart rhythm (Holter) monitoring.

\begin{tabular}{cccc}
\hline & Landiolol & Placebo & P \\
\hline No. of patients & 22 & 21 & 0.10 \\
Partial block & 6 & 2 & 0.32 \\
Negative T wave & 0 & 1 & 0.27 \\
Sinus arrhythmia & 8 & 5 & $* 0.03$ \\
Atrial fibrillation & 1 & 6 & $*$ \\
\hline
\end{tabular}

$$
{ }^{*} \mathrm{P}<0.05
$$


Table 3. Heart rate (Holter) monitoring (beats/min).

\begin{tabular}{cccc}
\hline & Landiolol & Placebo & P \\
\hline Preoperative & $70.2 \pm 14.6$ & $73.3 \pm 12.2$ & 0.23 \\
Postoperative-24 hour & $78.9 \pm 11.3$ & $76.6 \pm 9.9$ & 0.52 \\
$24-48$ hour & $80.0 \pm 12.6$ & $82.8 \pm 10.7$ & 0.48 \\
48 - 72 hour & $82.6 \pm 11.3$ & $83.3 \pm 7.7$ & 0.87 \\
\hline
\end{tabular}

\section{Discussion}

This study has two main findings. First, landiolol decreases the incidence of AF for up to 72 hours after surgery. In this study, AF onset occurred an average of 2.7 days after surgery. This result is, therefore, similar to the findings of previous studies, which have stated that AF is prone to occur from 2 or 3 days after surgery [6] [7]. The second key finding of this study is that low doses of landiolol, such as those used in this study, rarely result in adverse reactions, such as excessive reductions in heart rate and blood pressure.

The present study demonstrated that landiolol decreases the incidence of intraoperative AF with only a small dose. Studies of a similar-blocker, metoprolol, have also shown a significant reduction in the incidence of AF [8]. However, metoprolol was orally administered from preoperatively to 4 days postoperatively, which is less convenient than the administration of landiolol in the present study. Specifically, landiolol was administered only intraoperatively in the present study, offering both a shorter duration and the ability to lower the incidence of AF through management by the anesthesiologist alone. Moreover, since only a small dose of landiolol was given, obvious side effects did not develop. Even if an adverse reaction had occurred, landiolol has a short half-life (4 minutes) as compared with metoprolol (2.8 hours), is well-regulated, and is easy to use.

The mechanism of AF onset after thoracic surgery remains unclear, although the origin of AF and its association with the pulmonary vein have both been demonstrated. In AF, frequent local excitation (premature atrial contraction) is the trigger for the initial formation and onset of reentry arrhythmia. Haissaguerre et al. reported that the aberrant excitation that triggers AF mostly originates in the atrial muscle in the pulmonary veins [9]. Meanwhile, based on the results of electrophysiological testing, Jaiss et al. found that premature atrial contraction typically originates at the entrance to the pulmonary veins [10]. The complex network of cardiac muscles at the junction of the pulmonary vein and left atrium means that delayed conduction and reentry is prone to occur at this site [11]. Further, stretch stimulation accompanying increased pulmonary venous pressure may enhance motility via stretch-activated channels [12]. During lung resection, pulmonary vein dissection is believed to induce AF.

Although it is not entirely clear why handling of the pulmonary vein triggers AF, our findings show a strong correlation between AF and thoracic surgery. Indeed, AF occurred in $17 \%$ of our patients, including $28 \%$ of those in the control group and $5 \%$ of those in the landiolol group $(\mathrm{P}=0.03)$. These findings indicate that the incidence of AF was significantly inhibited in the group of patients who received landiolol.

Our findings demonstrate that administration of landiolol during surgery decreased the incidence of AF following lung resection. Although-blockers, such as landiolol, are known to reduce perioperative increases in sympathetic nervous system (SNS) activity and reduce AF onset, the underlying mechanisms of these changes remain unclear. Further research is needed to determine the optimal dose of landiolol required to reduce AF onset.

Our study has the following limitations. First, the incidence of AF was greater than those in many previous reports. This was attributed to the high percentage of elderly patients in the present study, and in fact, the mean age of the seven patients who developed AF was 82 years. It is known that aging causes degenerative changes in atrial anatomy that are accompanied by a shorter atrial effective refractory period, longer sinoatrial and atrioventricular nodal conduction times, atrial stiffening, and splitting of the atrial excitation waveform by the pectinate trabeculae [13] [14]. The second limitation of our study was the small patient cohort. Further study is therefore necessary, and should include the assessment of landiolol administration in a large cohort of patients.

\section{Conclusion}

In conclusion, perioperative landiolol administration was found to be an effective means of reducing the incidence of atrial fibrillation after pulmonary lobectomy. 


\section{References}

[1] Sakamoto, A., Kitazawa, M., Takamoto, S., et al., JL-KNIGHT Study Group (2012) Landiolol, an Ultra-Short-Acting $\beta_{1}$-Blocker, More Effectively Terminates Atrial Fibrillation than Diltiazem after Open Heart Surgery: Prospective, Multicenter, Randomized, Open-Label Study (JL-KNIGHT Study). Circulation Journal, 76, 1097-1101. http://dx.doi.org/10.1253/circj.CJ-11-1332

[2] Rena, O., Papalia, E., Oliaro, A., et al. (2001) Supraventricular Arrhythmias after Resection Surgery of the Lung. European Journal Cardio-Thoracic Surgery, 20, 688-693. http://dx.doi.org/10.1016/S1010-7940(01)00890-9

[3] Cox, J.L. (1993) A Perspective of Postoperative Atrial Fibrillation in Cardiac Operations. Annals of Thoracic Surgery, 56, 405-409. http://dx.doi.org/10.1016/0003-4975(93)90871-E

[4] Polanczyk, C.A., Goldman, L., Marcantonio, E.R., et al. (1998) Supraventricular Arrhythmia in Patients Having Noncardiac Surgery. Clinical Correlates and Effect on Length of Stay. Annals of Internal Medicine, 129, 279-285. http://dx.doi.org/10.7326/0003-4819-129-4-199808150-00003

[5] Ito, K., Nozaki, N., Sakamoto, R., et al. (2014) Safety of Landiolol Infusion in Patients Undergoing Lung Resection. Open Journal of Anesthesiology, 8, 183-190. http://dx.doi.org/10.4236/ojanes.2014.48026

[6] Rena, O., Papalia, E., Oliaro, A., et al. (2001) Supraventricular Arrhythmias after Resection Surgery of the Lung. European Journal Cardio-Thoracic Surgery, 20, 688-693. http://dx.doi.org/10.1016/S1010-7940(01)00890-9

[7] Jacobsen, C.J., Aoren, B., Peter, A., et al. (1997) Perioperative Metoprolol Reduces the Frequency of Atrial Fibrillation after Thoracotomy for Lung Resection. Journal of Cardiothoracic and Vascular Anesthesia, 11, 746-751. http://dx.doi.org/10.1016/S1053-0770(97)90169-5

[8] Gomez-Caro, A., Mordieellos, F.J., Ausin, P., et al. (2006) Risk Factors for Atrial Fibrillation after Thoracic Surgery. Archivos de Bronconeumología, 42, 9-13. http://dx.doi.org/10.1016/S1579-2129(06)60107-9

[9] Haissaguerre, M., Jais, P., Shah, D.C., et al. (1998) Spontaneous Pulmonary Veins. New England Journal of Medicine, 339, 659-666.

[10] Jaiss, P., Haissaguerre, M., Dipen, S., et al. (1997) A Focal Source of Atrial Fibrillation Treated by Discrete Radiofrequency Ablation. Circulation, 95, 572-576. http://dx.doi.org/10.1161/01.CIR.95.3.572

[11] Hocini, M., Ho, S.Y., Kawara, T., et al. (2002) Electrical Conduction in Canine Pulmonary Veins. Electrophysiological and Automic Correlation. Circulation, 105, 2442-2448. http://dx.doi.org/10.1161/01.CIR.0000016062.80020.11

[12] Bode, F., Katchman, A., Woosley, R.L., et al. (2000) Gadolinium Decrease Stretch-Induced Vulnerability to Atrial Fibrillation. Circulation, 101, 2200-2205. http://dx.doi.org/10.1161/01.CIR.101.18.2200

[13] Amar, D., Roistacher, N., Rusch, V.W., et al. (2000) Effects of Diltiazem Prophylaxis on the Incidence and Clinical Outcome of Atrial Arrhythmias after Thoracic Surgery. Journal of Thoracic and Cardiovascular Surgery, 120, 790798. http://dx.doi.org/10.1067/mtc.2000.109538

[14] Misier, A.R., Opthof, T., van Hemel, N.M., et al. (1992) Increased Dispersion of Refractoriness in Patients with Indiopathic Paroxysmal Atrial Fibrillation. Journal of the American College of Cardiology, 19, 1531-1535. http://dx.doi.org/10.1016/0735-1097(92)90614-S

\section{Scientific Research Publishing}

\section{Submit or recommend next manuscript to SCIRP and we will provide best service for you:}

Accepting pre-submission inquiries through Email, Facebook, LinkedIn, Twitter, etc.

A wide selection of journals (inclusive of 9 subjects, more than 200 journals)

Providing 24-hour high-quality service

User-friendly online submission system

Fair and swift peer-review system

Efficient typesetting and proofreading procedure

Display of the result of downloads and visits, as well as the number of cited articles

Maximum dissemination of your research work

Submit your manuscript at: http://papersubmission.scirp.org/ 